\title{
Metastable hydrogen absorption in ejecta close to $\eta$ Carinae
}

\author{
S. Johansson ${ }^{1}$, T. R. Gull ${ }^{2}$, H. Hartman ${ }^{1}$, and V. S. Letokhov ${ }^{1,3}$ \\ ${ }^{1}$ Lund Observatory, Lund University, PO Box 43, 22100 Lund, Sweden \\ e-mail: [sveneric.johansson; henrik.hartman]@astro.lu.se \\ 2 Code 667, Goddard Space Flight Center, Greenbelt, MD, USA \\ e-mail: theodore.r.gull@nasa.gov \\ 3 Institute of Spectroscopy, Russian Academy of Sciences, Troitsk, Moscow region, 142190, Russia \\ e-mail: vladilen.letokhov@astro.lu.se
}

Received 29 April 2004 / Accepted 1 February 2005

\begin{abstract}
Spectroscopy with the high spatial resolution of the Hubble Space Telescope (HST) reveals narrow absorption in the hydrogen Balmer lines in spectra of Eta Carinae and the nearby nebular-scattered starlight. While hydrogen Balmer absorption lines are seen in stellar photospheres and winds, we are not aware of such being seen in galactic nebulae. This exceptional case is caused by intense stellar UV radiation acting on high-density neutral clumps of gas in the close vicinity of the central source. The interaction of the UV radiation with hydrogen results in photo-ionization and photo-excitation leading to a nonequilibrium population of the metastable $2 \mathrm{~s}{ }^{2} \mathrm{~S}$ level. This occurs throughout the equatorial region surrounding $\eta$ Carinae in sufficient quantity to produce strong narrow absorption on top of the broad P Cygni emission profile. This absorption can be considered to be a probe of the very non-uniform ejecta in the disk region surrounding $\eta$ Carinae.
\end{abstract}

Key words. atomic processes - line: formation - line: profiles - H II regions - radiation mechanisms: non-thermal stars: individual: $\eta$ Carinae

\section{Introduction}

Eta Carinae $\left(\eta\right.$ Car) is a very massive $\left(>100 M_{\odot}\right)$, variable star emitting $5 \times 10^{6} L_{\odot}$, and is considered a prototype for the Luminous Blue Variables (LBV's) (Davidson \& Humphreys 1997). The star itself, most likely a binary (Damineli 1996; Pittard \& Corcoran 2002), is surrounded by massive, mostly neutral material in the form of the Homunculus, ejected during the Great Eruption of the 1840's. The rapidly expanding bipolar-lobed structure now extends 18 arcsec across the sky and is tilted from line of sight by 41 degrees (Davidson et al. 2001). Between these lobes is a more slowly moving skirt, that extends from the very near vicinity of $\eta$ Car radially outward. Three nearly pointlike knots, called the Weigelt blobs B, C and $\mathrm{D}$, were first observed by speckle interferometry (Weigelt \& Ebersberger 1986). The Weigelt blobs were later identified with HST's Faint Object Spectrograph to be emission nebulae (Davidson et al. 1995). More recently Smith et al. (2004) identified several more knots that form a complete chain surrounding $\eta$ Car, but appear to be bright reflection knots. All these knots are close to the central source (about 0.1 to 0.3 arcsec or a projected distance of 230 to $700 \mathrm{AU}$ at the adopted distance of $2300 \mathrm{pc}$ to the star).

Beginning in 1998, a series of observations have been accomplished with the HST's Space Telescope Imaging Spectrograph (STIS) taking advantage of its very high angular resolution (Gull et al. 2001, see several HST Programs with Davidson or Gull as Principal Investigator). From 1640-10300 $\AA$, CCD spectra of $\eta$ Car's central source, and thin slices of the Homunculus, were obtained with a $52^{\prime \prime} \times 0$.' 1 aperture and with a spectral resolving power $R=\lambda / \Delta \lambda \sim 7000$. The observations reveal much detailed information on the structure and the kinematics of the ejecta, plus temporal variations during the 5.54-year period first noted by Damineli (1996). While the Homunculus is primarily a reflection nebula centered on $\eta$ Car, its interior surface is revealed in the local emission of singly-ionized metals such as [Ni II], and [Fe II] (Davidson et al. 2001). Interior to the Homunculus, and also centered upon $\eta$ Car is the Little Homunculus (Ishibashi et al. 2003), an ionized bi-lobed structure that extends about two arc seconds above and below. It, too, has an associated skirt revealed by measurements of nebular emission line velocities and proper motion. The Weigelt blobs are associated with this skirt structure and consistent with the Little Homunculus being created by a lesser eruption in the 1890's (Smith et al. 2003). The skirt region therefore contains many individual ejecta moving at various velocities, produced by the two eruptions, and likely other events.

In the present paper we discuss the observation of narrow absorption components superimposed on the P Cygni profiles of Balmer lines in HST/STIS spectra of the central source and ejecta. We present an explanation based upon the double role 

$\eta$ Carinae
$\mathrm{H} \alpha$ profile

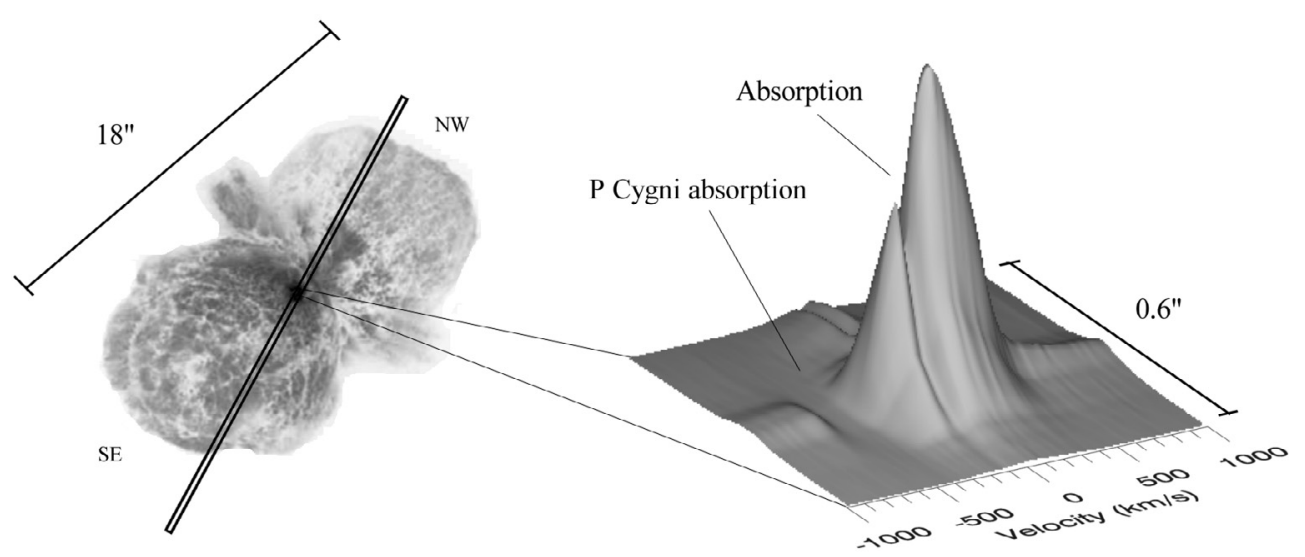

Fig. 1. Schematic picture of the HST/STIS observation. To the left the slit position is superimposed on a WFPC 2 image of $\eta$ Car and the Homunculus (Morse et al. 1998). To the right is a three-dimensional image of a portion of the Balmer- $\alpha$ spectrum with intensity being the elevation. The stellar line profile is P-Cygni type in shape, where the large valley feature (P Cygni) is the absorption of the massive stellar wind moving in the direction of the observer. A narrow absorption feature closer to the center originates from metastable hydrogen, $2 \mathrm{~s}^{2} \mathrm{~S}$, located in extended low-density ejecta.
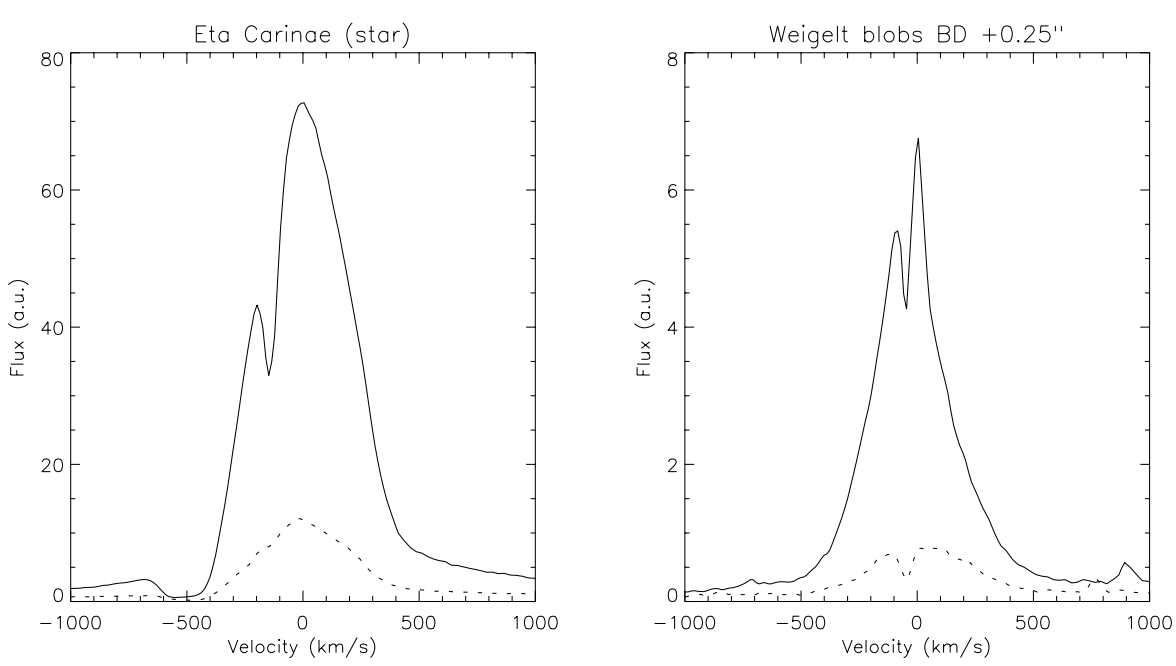

Fig. 2. Balmer line profiles, Balmer- $\alpha$ (solid curve) and Balmer- $\beta$ (dashed curve), in the line of sight to the central source (left panel) and to the Weigelt blobs BD (right panel). A narrow absorption component appears at different velocities for the central source and the blobs.

of stellar radiation: first, the starlight produces metastable hydrogen in the 2 s state in relatively cool, weakly ionized ejecta. Second, the extended, wind-driven atmosphere of the central source provides intense, broad Balmer emission line radiation. The interaction between the Balmer emission and the metastable hydrogen inside the ejecta results in narrow absorption superimposed on the wide Balmer lines. The broad P-Cygni Balmer emission profiles have been investigated by Davidson et al. (2005).

\section{Observational data}

The radiation from the central source and its immediate vicinity (Figs. 1 and 2) exhibits narrow Balmer line absorption superimposed upon the emission portion of the P-Cygni Balmer line profiles. These narrow lines are present beginning with Balmer- $\alpha$ and continuing with several higher members of the Balmer series. In Fig. 1, we show an example of a long slit STIS spectrum centered upon Balmer- $\alpha$ with the 0.1 wide aperture position superimposed upon a WFPC2 image of $\eta$ Car and the Homunculus (Morse et al. 1998). In heliocentric velocity space, the P-Cygni profile has a broad blue-shifted absorption extending from -350 to $-550 \mathrm{~km} \mathrm{~s}^{-1}$ and a broad emission with a red tail. Superimposed on the direct stellar spectrum is a narrow absorption at approximately $-150 \mathrm{~km} \mathrm{~s}^{-1}$. An absorption component at the velocity $-146 \mathrm{~km} \mathrm{~s}^{-1}$ is also seen in multiple narrow lines of singly-ionized iron-group elements in the UV STIS high-dispersion echelle spectra (Gull et al. 2004). As shown in Fig. 1 the long aperture, oriented at -28 degrees position angle, extends from the foreground Southeast (SE) lobe (bottom) across $\eta$ Car and the background Northwest (NW) lobe, with the extended skirt region. To the NW, the Balmer- $\alpha$ absorption is partially filled in by nebular emission from the Weigelt blobs B and D, plus the Little Homunculus. 
Within a resolution width $\left(0{ }^{\prime} 1\right.$ or 230 AU) of HST, a second velocity component appears at $-45 \mathrm{~km} \mathrm{~s}^{-1}$. This corresponds very closely to $-46 \mathrm{~km} \mathrm{~s}^{-1}$ measures of UV nebular emission lines from Weigelt blobs B and D. Multiple slit spectra of Balmer- $\alpha$ recorded by STIS in March 2000 for mapping the Little Homunculus (Ishibashi et al. 2003), show that the Balmer- $\alpha$ absorption is found against most of the skirt region.

In Fig. 2 we show the line profiles of Balmer- $\alpha$ and Balmer- $\beta$ in spectra centered on the star and on the Weigelt blobs $\mathrm{B}$ and $\mathrm{D}$ (note the different intensity scales) at position angle -28 degrees. The emission profiles are very different for each position due to scattered P-Cygni stellar profiles and nebular emission in the line of sight. A narrow absorption component appears at $-150 \mathrm{~km} \mathrm{~s}^{-1}$ on the star, but to the NW the narrow component abruptly shifts to $-45 \mathrm{~km} \mathrm{~s}^{-1}$, in excellent agreement with the projected blueshifts for blobs B and D (Davidson et al. 1997). The broad P-Cygni profile, centered on $-500 \mathrm{~km} \mathrm{~s}^{-1}$ is clearly present on the star, but is significantly less pronounced in the nebular-scattered light.

On the star, the time behaviour of the narrow Balmer- $\alpha$ absorption component at $-150 \mathrm{~km} \mathrm{~s}^{-1}$ around the spectroscopic event in the summer of 2003 is shown in Fig. 3. The equivalent width, normalized to the largest value, in the Balmer- $\alpha$ emission profile seen in the spectrum in line-of-sight to the central source is plotted as a function of time at seven different occasions. The curve shows three distinct regions: a small amount of absorption before the event ( $\approx-40$ to -20 days), increasing absorption in the beginning of the event $(\approx-20$ to +30 days $)$ and a slow decrease after the maximum ( $>+30$ days). A plausible interpretation of these three regions is given in Sect. 6 . The response of the ejecta (Weigelt blobs B and D) to changes in the UV flux from the star, is represented in Fig. 3 by the average flux of the two fluorescent Fe II lines $\lambda \lambda 2507 / 2509$ in the blob spectra. These lines, excited by HLy $\alpha$, start to decline by 40 days before the X-ray drop. The decline is steepest when the Balmer- $\alpha$ absorption increases most rapidly. The relative strength for the narrow absorption component of Balmer- $\alpha$ seen in the blob spectrum in Fig. 2 is difficult to measure reliably, but it shows a similar time behaviour as the absorption along the line-of-sight to the star.

\section{Ways of populating the $\mathrm{H}\left(2 \mathrm{~s}^{2} \mathrm{~S}\right)$ state by $\eta$ Car radiation}

The narrow Balmer absorption lines can be explained by a simple model. The nebular structure immediately surrounding Eta Carinae is very nonuniform, consisting of many very dense clumps of neutral, or partially ionized, gas in a sea of dense ionized gas, not the classical Strömgren H II region. The Weigelt blobs B, C and D are large examples of these clumps. Each clump is neutral, being shielded by H II and Fe II. Lyman radiation penetrates these neutral clumps, leading to Ly $\alpha$-pumped Fe II emission (Johansson \& Letokhov 2003), especially during the broad spectroscopic maximum. The UV flux of an O-star companion is necessary to support the ionization structure of the gas surrounding these blobs during the maximum (Verner et al. 2005). Characteristic densities derived of the Weigelt blobs in the models were approximately $10^{8} \mathrm{~cm}^{-3}$, much denser

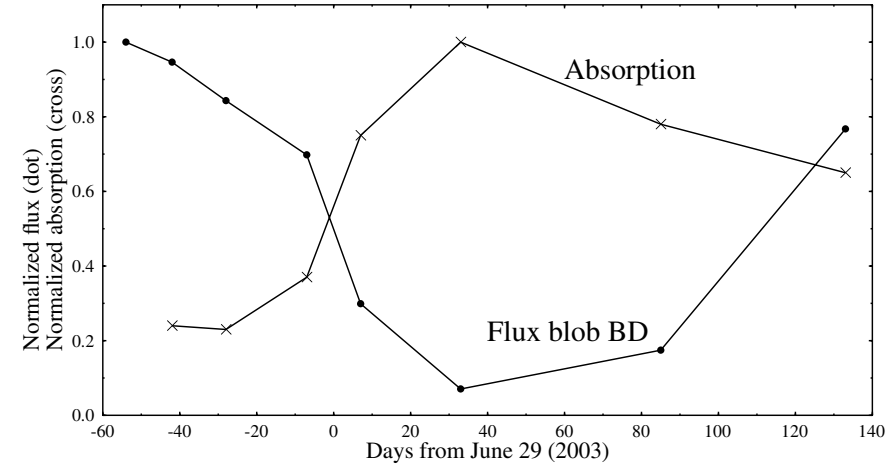

Fig. 3. Changes with time of Balmer- $\alpha$ narrow-line absorption $(x)$ and Ly- $\alpha$ pumped emission $(\bullet$ ) across the spectroscopic event (2003.5) of $\eta$ Car. The Balmer- $\alpha$ narrow-line absorption was measured in the lineof-sight of the central star (see Fig. 2a). The response of the ejecta to the stellar UV radiation is represented by the average flux of two Ly $\alpha$ pumped fluorescence line of Fe II ( $\lambda \lambda$ 2507/2509) in the spectrum of the Weigelt blobs B and D $(\bullet)$. Both curves are normalized to the largest values, respectively.

than the 10 to $10^{3} \mathrm{~cm}^{-3}$ for most $\mathrm{H}$ II regions. Throughout the skirt region between the bipolar lobes of the Homunculus these neutral clumps are surrounded by an extended ionized hydrogen sea, with many $\mathrm{H} \mathrm{II/H} \mathrm{I} \mathrm{zones.} \mathrm{Within} \mathrm{this} \mathrm{rather} \mathrm{non-}$ classical Strömgren region, continuous photoionization, radiative recombination and photoexcitation processes take place leading to population of the metastable $2 \mathrm{~s}^{2} \mathrm{~S}$ state of hydrogen. We restrict ourselves to the $\mathrm{H}$ II zone extending out to several thousand AU. In this H II region, we consider the most important processes: (1) photoionization followed by recombination (Fig. 4a); and (2) photoexcitation (Fig. 4b).

In $\mathrm{H}$ II regions the metastable level $2 \mathrm{~s}{ }^{2} \mathrm{~S}$ of $\mathrm{H}$ I can be populated by the following two mechanisms: (1) photoionization of $\mathrm{HI}$ by the Lyman continuum, followed by recombination to the $2 \mathrm{~s}^{2} \mathrm{~S}$ level; and (2) photoexcitation of the levels $n \mathrm{p}{ }^{2} \mathrm{P}$ $(n \geq 3)$ by the $\mathrm{Ly} \beta, \gamma, \delta$ radiation from $\eta$ Car with subsequent radiative decay to the $2{ }^{2} \mathrm{~S}$ level. Both of these mechanisms provide comparable population rates of the $2 \mathrm{~s}^{2} \mathrm{~S}$ level within the Strömgren boundary, wherein hydrogen is ionized with very small amounts of neutral hydrogen.

However, the characteristic times for these two mechanisms are very different. The photoexcitation (with rate $W_{\text {exc }}$ ) is directly correlated to the intensity of $\operatorname{Ly} \beta$. But, the photoionization/recombination mechanism is not correlated to the intensity of the Lyman continuum, $\mathrm{Ly}_{\mathrm{c}}$; it is delayed by the time of recombination, i.e. the inverse recombination rate $W_{\mathrm{ph}}^{-1}$. This difference is distinguishable during the spectroscopic event in $\eta$ Car.

In Fig. 4b, a schematic diagram is presented of the quantum transitions that are relevant for the population of the $2 \mathrm{~s}^{2} \mathrm{~S}$ level by the scheme $1 \mathrm{~s} \rightarrow n \mathrm{p}^{2} \mathrm{P} \rightarrow 2 \mathrm{~s}^{2} \mathrm{~S}$ together with the rates of the corresponding radiative transitions. For the sake of brevity, we will restrict ourselves to photoexcitation of the $2{ }^{2} \mathrm{~S}$ level by $\mathrm{Ly} \beta$ radiation via the $3 \mathrm{p}^{2} \mathrm{P}$ level, which populates this state with a branching fraction $B F=A_{32} /\left(A_{31}+A_{32}\right)=0.15$. In the rarefied nebular component, the collisional relaxation of the level $2 \mathrm{~s}^{2} \mathrm{~S}$ can be neglected as the rate of the two-photon decay 


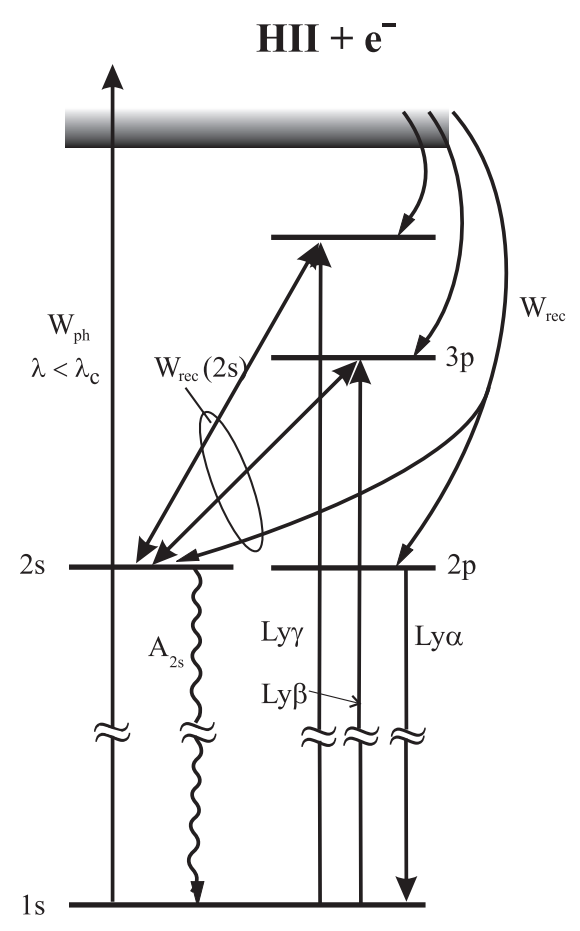

A.

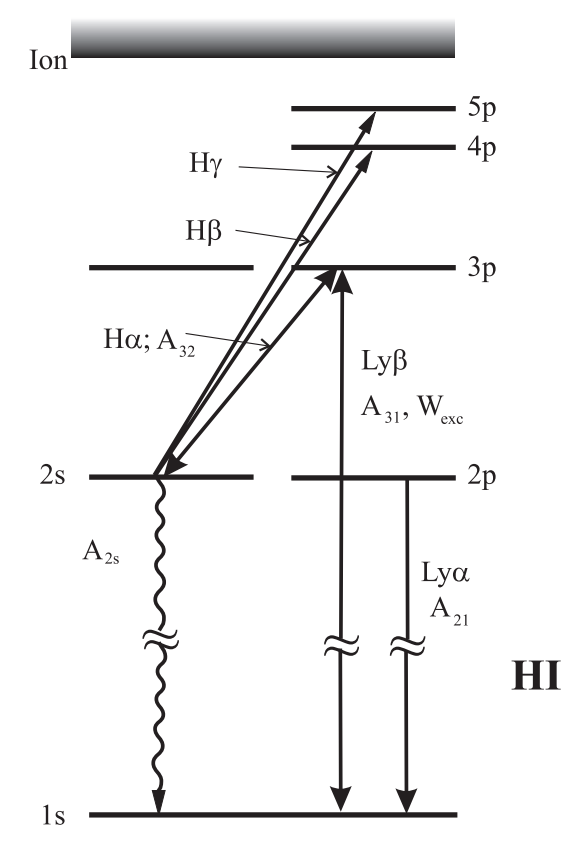

B.

Fig. 4. Mechanisms for populating the metastable $2 \mathrm{~s}^{2} \mathrm{~S}$ state of $\mathrm{H}$ I by stellar radiation from $\eta$ Car: a) excitation due to recombination preceded by photo-ionization by the Lyman continuum radiation; b) resonant excitation by the Lyman lines, Ly $\beta$, Ly $\gamma$, etc. Only energy levels of relevance are included in the diagram.

$2 \mathrm{~s} \rightarrow 1 \mathrm{~s}, A_{2 \mathrm{~s}}=8.2 \mathrm{~s}^{-1}$, is much higher than the rate of any collision. The steady-state population of the $2 \mathrm{~s}^{2} \mathrm{~S}$ level due to photoexcitation by $\operatorname{Ly} \beta$ is defined by the expression:

$N_{2}^{\mathrm{exc}} \simeq N_{\mathrm{H}} \frac{W_{\mathrm{rec}}}{W_{\mathrm{ph}}} \frac{W_{\mathrm{exc}}}{A_{2 s}} B F$,

where we account for the fact that $W_{\text {exc }} \ll A_{2 \text { s }}$ everywhere except very near to the star (see Sect. 4), and that the ground state population of hydrogen $N_{1}=N_{\mathrm{H}}\left(W_{\mathrm{rec}} / W_{\mathrm{ph}}\right) . N_{\mathrm{H}}$ is the total density of hydrogen.

It is immediately evident from Eq. (1) that if the hydrogen distribution is homogeneous in the nebular component, the population of the metastable $2 \mathrm{~s}{ }^{2} \mathrm{~S}$ state is independent of the distance $R$ from the central star since both the photoexcitation and photoionization rates, $W_{\mathrm{exc}}$ and $W_{\mathrm{ph}}$, are proportional to $1 / R^{2}$. In the following we estimate the ratio $\frac{W_{\mathrm{exc}}}{W_{\mathrm{ph}}}$ for $\mathrm{HI}$, which will be taken as a constant in the following discussion. The photoexcitation rate, $W_{\text {exc }}$, of the $\mathrm{H}$ atoms may be represented in the form:

$W_{\mathrm{exc}}=\frac{A_{31}}{(2 \pi)^{2}} \frac{g_{3}}{g_{1}}\left(\frac{r_{\mathrm{s}}}{2 R}\right)^{2}\left(\mathrm{e}^{\frac{h \nu_{13}}{\mathrm{k}_{\mathrm{eff}}}}-1\right)^{-1}$,

where $T_{\text {eff }}$ and $r_{\mathrm{s}}$ are the photospheric temperature and stellar radius of $\eta$ Car. It follows from Eq. (2) that at $T_{\text {eff }} \cong 30000 \mathrm{~K}$, $r_{\mathrm{s}} \cong 1.5 \times 10^{13} \mathrm{~cm}$ (Davidson \& Humphreys 1997) and $A_{31} \cong$ $10^{8} \mathrm{~s}^{-1}$, the condition $A_{2 \mathrm{~s}} \simeq W_{\mathrm{exc}}$ is satisfied at a value of the distance $R \simeq 200 r_{\mathrm{s}}=3 \times 10^{15} \mathrm{~cm}$. Thus, the condition $W_{\mathrm{exc}}<$ $A_{2 \mathrm{~s}}$ is valid at distances $R>3 \times 10^{15} \mathrm{~cm}$ from the star, i.e. in the low-density extended ejecta expelled by the central star.
The rate of photoionization of $\mathrm{HI}$ from the ground state (Fig. 4a) can be estimated using the expression

$$
\begin{aligned}
W_{\mathrm{ph}} & \simeq\left(\frac{r_{\mathrm{s}}}{2 R}\right)^{2} \int_{v_{c}}^{\infty} \sigma_{\mathrm{ph}}(v) P(v, T) \mathrm{d} v \\
& \simeq\left(\frac{r_{\mathrm{s}}}{2 R}\right)^{2}\left\langle\sigma_{\mathrm{ph}}\right\rangle\langle P(v, T)\rangle \Delta v_{\mathrm{ph}},
\end{aligned}
$$

where $P(v, T)\left(\frac{r_{\mathrm{s}}}{2 R}\right)^{2}$ is the spectral intensity of $\operatorname{Ly} \beta$ at a distance $R$ from the photosphere of $\eta$ Car. For simplicity we take the average values $\left\langle\sigma_{\mathrm{ph}}\right\rangle$ and $\langle P(v, T)\rangle$ in the spectral band $h \Delta v_{\mathrm{ph}} \simeq 3 \mathrm{eV}$ above the photoionization limit $h v_{\mathrm{c}}$. The frequency dependence on $P(v, T)$ and $\sigma_{\mathrm{ph}}$ above the ionization limit of $\mathrm{HI}$ implies a reduction by a factor of 2-3 of these parameters in the $3 \mathrm{eV}$ spectral interval 13.6 to $16.6 \mathrm{eV}$, which we use for the estimate of their average values $\langle P(v, T)\rangle$ and $\left\langle\sigma_{\mathrm{ph}}\right\rangle$. In that case, one can take $\langle P(v, T)\rangle \simeq 7 \times$ $10^{7}$ (photons $/ \mathrm{cm}^{2} \mathrm{~s} \mathrm{~Hz} \mathrm{sr}$ ), or the ionizing Lyman continuum radiation flux on the surface of $\eta$ Car: $\Phi_{\mathrm{ph}}=\langle P(v, T)\rangle \Delta v_{\mathrm{ph}} \simeq 3 \times$ $10^{22}$ (photons $/ \mathrm{cm}^{2} \mathrm{~s} \mathrm{sr}$ ).

Using a general expression for $W_{\mathrm{exc}}$ and Eq. (3) for $W_{\mathrm{ph}}$ we get

$\frac{W_{\mathrm{exc}}}{W_{\mathrm{ph}}} \simeq \frac{\sigma_{13}}{\left\langle\sigma_{\mathrm{ph}}\right\rangle} \frac{\Delta v_{13}}{\Delta v_{\mathrm{ph}}} \frac{P\left(v_{13}, T\right)}{\langle P(v, T)\rangle}$.

The ratio between the spectral intensities of the black body radiation $P(v, T)$ of the central source at $30000 \mathrm{~K}$ causing the photoexcitation by $\operatorname{Ly} \beta$ and the photoionization radiation from 
$\eta$ Car can be estimated to be around 3. Using the expression for $\sigma_{13}$, one can thus reduce Eq. (4) to the simple expression

$\frac{W_{\mathrm{exc}}}{W_{\mathrm{ph}}} \simeq \frac{\lambda_{13}^{2}}{2\left\langle\sigma_{\mathrm{ph}}\right\rangle} \frac{A_{31}}{2 \pi \Delta \nu_{\mathrm{ph}}} \frac{g_{3}}{g_{1}}$.

For this case of $\operatorname{Ly} \beta$ excitation and average values of $\left\langle\sigma_{\mathrm{ph}}\right\rangle \simeq$ $3 \times 10^{-18} \mathrm{~cm}^{2}$ and $\Delta v_{\mathrm{ph}} \simeq 8 \times 10^{14} \mathrm{~Hz}$ (from the $3 \mathrm{eV}$ width), we get a simple, but important, estimate: $W_{\mathrm{exc}} \cong W_{\mathrm{ph}}\left(v>v_{\mathrm{c}}\right)$. Hence, we have the following expression for the density of metastable atoms expressed above in Eq. (1):

$N_{2}^{\mathrm{exc}} \simeq N_{\mathrm{H}}^{2} \frac{\alpha B F}{A_{2 \mathrm{~s}}} \frac{W_{\mathrm{exc}}}{W_{\mathrm{ph}}} \simeq 0.5 \times 10^{-14} \cdot N_{\mathrm{H}}^{2} \quad\left(\mathrm{~cm}^{-3}\right)$

where we have inserted the values of $A_{2 \mathrm{~s}}=8.2 \mathrm{~s}^{-1}, \alpha \cong 3 \times$ $10^{-13} \mathrm{~cm}^{3} / \mathrm{s}$, and the branching fraction $B F \cong 0.15$. For example, at $N_{\mathrm{H}} \simeq 10^{4}-10^{5} \mathrm{~cm}^{3}$ the quantity $N_{2}^{\text {exc }}$ is approximately $10^{-6}-10^{-5} \mathrm{~cm}^{-3}$.

We have obtained an estimate (Eq. (5)) of the ratio between the rates of photoexcitation of $\mathrm{H}$ I by $\mathrm{Ly} \beta$ radiation and of photoionization $W_{\mathrm{ph}}$, from which it follows that they are of the same order of magnitude. Each photoionization event leads to recombination to the level $2 \mathrm{~s}^{2} \mathrm{~S}$ (Fig. 4a) with the coefficient $\alpha(2 \mathrm{~s})=\gamma \alpha(1 \mathrm{~s})(\gamma \cong 0.06)$, and each $3 \mathrm{p}$-level photoexcitation event by $\operatorname{Ly} \beta$ leads to the population of the level $2 \mathrm{~s}{ }^{2} \mathrm{~S}$ with a branching fraction of 0.15 . Therefore, the steady-state population of the $2 \mathrm{~s}^{2} \mathrm{~S}$ level within the H II region $\left(R<R_{\mathrm{Str}}\right)$ is defined by the relation

$\frac{N_{2}^{\mathrm{exc}}}{N_{2}^{\mathrm{rec}}} \simeq \frac{B F W_{\mathrm{exc}}}{\gamma W_{\mathrm{ph}}} \simeq 2.5 \frac{W_{\mathrm{exc}}}{W_{\mathrm{ph}}} \simeq 2.5$

since $W_{\text {exc }}(\mathrm{Ly} \beta) \cong W_{\mathrm{ph}}\left(v>v_{\mathrm{c}}\right)$ according to Eq. (5).

In spite of a low fraction of neutral hydrogen and a low population of the metastable $2 \mathrm{~s}$ state it is shown in Sect. 5 that the population of the $2 \mathrm{~s}^{2} \mathrm{~S}$ level by these mechanisms yields an optical depth, which is sufficient for absorption in the transitions Balmer- $\alpha$, Balmer- $\beta$, Balmer- $\gamma$, through Balmer- $\delta$. However, the population of the $2 \mathrm{~s}$ state will be negligible in the near vicinity of $\eta$ Car because of depletion by photoionization of this state provided by stellar radiation.

\section{Photoionization depletion of the $\mathrm{HI} 2 \mathrm{~s}^{2} \mathrm{~S}$ state}

The metastable state $2 \mathrm{~s}^{2} \mathrm{~S}$ has a very low two-photon probability (the radiative lifetime is $0.12 \mathrm{~s}$ ) but it can be emptied through photoionization, photoionization depletion, by photospheric radiation from the star. This photoionization depletion of the $2 \mathrm{~s}$ state is significant if the rate of photoionization $W_{\mathrm{ph}}^{2 \mathrm{~s}}$ is comparable or higher than the rate of two-photon decay $A_{2 \mathrm{~s}}=8.2 \mathrm{~s}^{-1}$. Let us define the photoionization depletion radius $R_{\mathrm{dep}}$ as that distance from the star where $W_{\mathrm{ph}}^{2 \mathrm{~s}}=A_{2 \mathrm{~s}}$.

The rate of photoionization of the metastable $2 \mathrm{~s}$ state $W_{\mathrm{ph}}^{2 \mathrm{~s}}$ is determined by an expression of the same type as Eq. (3):

$$
\begin{aligned}
W_{\mathrm{ph}}^{2 \mathrm{~s}} & =\left(\frac{r_{\mathrm{s}}}{2 R}\right)^{2} \int_{v_{c}-v_{2 \mathrm{~s}}}^{\infty} \sigma_{\mathrm{ph}}^{2 \mathrm{~s}}(v) P(v, T) \mathrm{d} v \\
& \simeq\left(\frac{r_{\mathrm{s}}}{2 R}\right)^{2}\left\langle\sigma_{\mathrm{ph}}^{2 \mathrm{~s}}\right\rangle\langle P(v, T)\rangle \Delta v_{\mathrm{ph}},
\end{aligned}
$$

where $h\left(v_{\mathrm{c}}-v_{2 \mathrm{~s}}\right)=3.4 \mathrm{eV}$ is the photoionization energy and $\sigma_{\mathrm{ph}}^{2 \mathrm{~s}}$ the photoionization cross-section of the $2 \mathrm{~s}$ state. The maximum value of $\sigma_{\mathrm{ph}}^{2 \mathrm{~s}}$ is about $2.5 \times 10^{-19} \mathrm{~cm}^{2}$. The spectral interval, $\Delta v_{\mathrm{ph}}$, effective for photoionization by photospheric radiation is about $3 \mathrm{eV}$, since the strong $1 / v^{3}$ dependence reduces the photoionization cross-section at higher frequencies and $\left\langle\sigma_{\mathrm{ph}}\right\rangle \approx$ $10^{-19} \mathrm{~cm}^{2}$. This estimation gives

$W_{\mathrm{ph}}^{2 \mathrm{~s}} \simeq 4 \times 10^{4}\left(\frac{r_{\mathrm{s}}}{2 R}\right)^{2} \quad\left(\mathrm{~s}^{-1}\right)$.

The rate of photoionization depletion will be equal to the rate of spontaneous two-photon decay $A_{2 \mathrm{~s}}$ at the distance $R_{\text {dep }}$ from the star, given by

$R_{\mathrm{dep}} \simeq r_{\mathrm{s}} \times 10^{2} \approx 10^{15}(\mathrm{~cm})$,

where $r_{\mathrm{s}}=1.5 \times 10^{13} \mathrm{~cm}$ is the stellar radius of $\eta$ Car. At distances $R>R_{\text {dep }}$ the two mechanisms considered above will provide the population of the $2 \mathrm{~s}$ state.

\section{Optical depth of Balmer line absorption}

We now estimate the length $L_{\text {abs }}$ over which the optical depth for the Balmer- $\alpha$ absorption is $D_{32} \simeq 1$. For a medium of length $L \cong L_{\text {abs }}$ we predict the observed Balmer nebular absorption. Setting $D_{32}=L_{\text {abs }} \sigma_{23}\left(N_{2}^{\text {exc }}+N_{2}^{\text {rec }}\right) \cong 1.4 \times L_{\text {abs }} \sigma_{23} N_{2}^{\text {exc }}$ to unity, sets the length to

$L_{\mathrm{abs}} \cong\left(1.4 \times \sigma_{23} N_{2}^{\mathrm{exc}}\right)^{-1} \cong 1.4 \times 10^{14} / \sigma_{23} N_{\mathrm{H}}^{2} \quad(\mathrm{~cm})$,

according to Eq. (6). Assuming that the narrow $\mathrm{H} \alpha$ absorption width $\Delta v_{23} \cong 5 \mathrm{~cm}^{-1}$ and $\sigma_{23} \cong 8 \times 10^{-14} \mathrm{~cm}^{2}$ we estimate $L_{\mathrm{abs}} \cong 1.4 \times 10^{27} \cdot N_{\mathrm{H}}^{-2} \mathrm{~cm}$. Figure 5 shows the variation of $L_{\mathrm{abs}}$ as a function of the total hydrogen density $N_{\mathrm{H}}$ in the absorbing nebular component of $\eta$ Car. The length $L_{\text {abs }}$ must be shorter than the radius $R_{\mathrm{Str}}$ of the Strömgren sphere, which contains $\mathrm{HII}$ and a very small amount of HI $\left(2 \mathrm{~s}{ }^{2} \mathrm{~S}\right)$. Therefore, we included in Fig. 5 the hydrogen density dependence of $R_{\text {Str }}$. Figure 5 also contains the limit values of $R_{\text {dep }}$, where the maximum value, $R_{\text {dep }}^{\max }$, corresponds to normal conditions in $\eta$ Car, and the minimum value, $R_{\mathrm{dep}}^{\mathrm{min}}$, refers to the conditions during the spectroscopic event. However, note the different meaning of $R_{\mathrm{dep}}$ and $L_{\mathrm{abs}}$.

The region around $\eta$ Car in terms of size $L$ and hydrogen density $N_{\mathrm{H}}$, where narrow-lined Balmer- $\alpha$ absorption can take place, is shown by the striped triangular sector in Fig. 5. However, the real absorption length cannot exceed the projected transversal size of the low-density extended equatorial media. For example, based on the data for an equatorial torus in the work by Morris et al. (1999), $L \cong 10^{18} \mathrm{~cm}$ and, accordingly, $N_{\mathrm{H}} \cong 3 \times 10^{4} \mathrm{~cm}^{-3}$.

As far as the order of magnitude is concerned, these estimations are valid for Balmer- $\beta$ and Balmer- $\gamma$ as well, considering the decrease of the $\operatorname{Ly} \gamma$ and $\operatorname{Ly} \delta$ intensities and the branching ratios Balmer- $\beta /$ Ly $\gamma$ and Balmer- $\gamma / \operatorname{Ly} \delta$. 


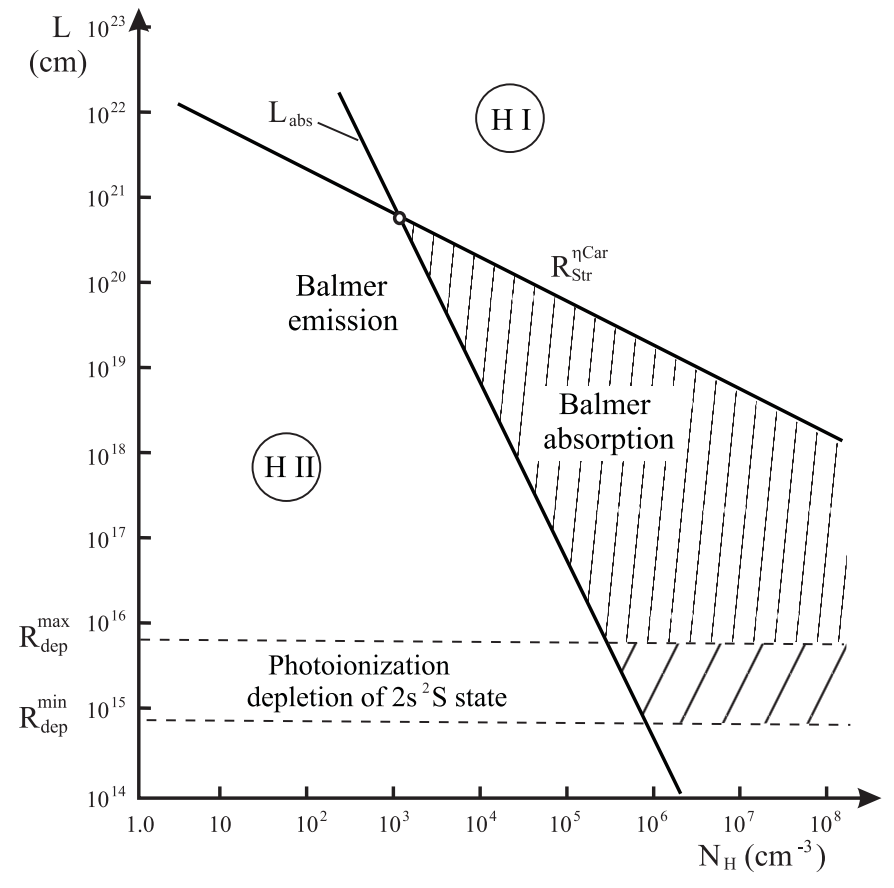

Fig. 5. The dependence of absorption length, $L$, on the total hydrogen density, $N_{\mathrm{H}}=N_{\mathrm{HI}}+N_{\mathrm{HII}}$. In a uniform density gas the fully-ionized HII region surrounding $\eta$ Car would lie below $R_{\mathrm{Str}}^{\eta \mathrm{Car}}$. However, clumps of neutral gas (H I) are surrounded by a sea of H II due to the ejection and wind phenomena. Balmer absorption by these neutral clumps can occur beyond a distance $L$ with a hydrogen density $N_{\mathrm{H}}$ that exceeds the critical absorption length $L_{\mathrm{abs}}=1.4 \times 10^{27} N_{\mathrm{H}}^{-2} \mathrm{~cm}<R_{\mathrm{Str}}-$ the shaded area labeled Balmer absorption. The lower limit of this critical density is defined by the photoionization depletion of the $2 \mathrm{~s}{ }^{2} \mathrm{~S}$ state. For $\eta$ Car this boundary shifts between the spectroscopic maximum $\left(R_{\mathrm{dep}}^{\max }\right)$, when UV radiation from the hot companion escapes the wind of the binary system, downward during the spectroscopic minimum $\left(R_{\mathrm{dep}}^{\min }\right)$.

\section{Discussion}

The time history of the observational data of Balmer absorption before and during the spectroscopic event in 2003 (Fig. 3) can be understood in terms of the photoprocesses discussed in the previous sections. As the steep curve in Fig. 3 shows the emission intensity of Ly $\alpha$ excited Fe II lines in blob B and $\mathrm{D}$, it also represents the stellar ionizing radiation reaching the blob and the absorbing media. We discuss the three consecutive phases of time variation of the Balmer- $\alpha$ absorption illustrated in Fig. 3: 1) a small amount of absorption before the spectral event ( $\approx-40$ to -20 days); 2 ) increasing absorption during the interval $(-20$ to +30$)$; and 3$)$ a slow decrease of absorption during the $(+30$ to +130$)$ interval. The Balmer- $\alpha$ absorption during these three periods is discussed in terms of three photo-processes: a) depletion of the $2 \mathrm{~s}{ }^{2} \mathrm{~S}$ state due to photoionization; b) resonant photoexcitation by $\operatorname{Ly} \beta$ with subsequent population of the $2 \mathrm{~s}^{2} \mathrm{~S}$ state; and c) photorecombination population of the $2 \mathrm{~s}^{2} \mathrm{~S}$ state.

Firstly, the small amount of absorption before the spectroscopic event, when a high level of stellar radiation is reaching the clumps, is explained by rapid photoionization depletion of the $2 \mathrm{~s}{ }^{2} \mathrm{~S}$ state, as discussed in Sect. 4. In Fig. 5 the maximum radius of photodepletion, $R_{\mathrm{dep}}^{\max }$ corresponds to a "normal" flux of stellar radiation, i.e. before the spectroscopic event. As the X-ray drop approaches, the increasing column density within the wind decreases the UV- ionizing radiation. Even at 40 days before the X-ray drop, the UV flux is decreasing, creating a smaller depletion radius, $R_{\text {dep }}^{\min }$ (see Fig. 5). Throughout the intermediate time zone ( -40 days to +40 days about the X-ray drop) the depletion radius is reduced to the range $R_{\text {dep }}^{\min }<R_{\text {dep }}<R_{\text {dep }}^{\max }$ resulting in a suppressed photoionization depletion. Absorption of the Balmer lines should increase, demonstrating qualitatively that the absorption regions are relatively close to the central source.

Secondly, the increase of absorption in the intermediate phase ( -20 to +30 days) in Fig. 5 can be qualitatively explained by suppression of photodepletion of the $2 \mathrm{~s}^{2} \mathrm{~S}$ state and contribution from photoexcitation of $\mathrm{Ly} \beta$ at a modest flux of stellar radiation and photorecombination to the $2 \mathrm{~s}{ }^{2} \mathrm{~S}$ state. At a low UV-ionization flux the main population of the $2 \mathrm{~s}^{2} \mathrm{~S}$ state is provided by recombination. The photoexcitation through the $1 \mathrm{~s}-3 \mathrm{p}-2 \mathrm{~s}$ pathway disappears, since the intensity of $\operatorname{Ly} \beta$ is weak during the spectroscopic event.

Thirdly, the existence of Balmer absorption during a significant reduction of starlight reaching the absorbing medium confirms the involvement of recombination in the population of the $2 \mathrm{~s}^{2} \mathrm{~S}$ state, since of the three processes photorecombination is the only one that can populate the excited states (including 2s) of hydrogen. The duration time for this mechanism is determined by the photorecombination time $\tau_{\text {rec }}$ :

$\tau_{\text {rec }}=\frac{1}{\alpha \cdot n_{\mathrm{e}}}(\mathrm{s})$, where $\alpha=\frac{3 \times 10^{-11}}{\sqrt{T}}\left(\mathrm{~cm}^{3} \mathrm{~s}^{-1}\right)$.

The electron density $n_{\mathrm{e}}=N_{\mathrm{H} \text { II }}$ assuming that hydrogen is the dominant ionized species. For $T \simeq 10^{4} \mathrm{~K}$ we get $\alpha \simeq 3 \times$ $10^{-13} \mathrm{~cm}^{3} \mathrm{~s}^{-1}$. During the period $t=\tau_{\text {rec }}$ the electron density $n_{\mathrm{e}}$ and recombination rate are reduced to the $1 / \mathrm{e}$ level. During the 90 days time interval ( +30 days, +120 days) in Fig. 3 the intensity of the Balmer- $\alpha$ absorption is reduced by only $30 \%$, which corresponds to a recombination time of about $2 \times 90=$ 180 days. According to Eq. (12) $\tau_{\text {rec }}=180$ days corresponds to $n_{\mathrm{e}} \simeq 2 \times 10^{5} \mathrm{~cm}^{-3}$. For the H II region $N_{\mathrm{H}} \approx n_{\mathrm{e}}$, and the absorbing medium is an extended ejecta with higher density than a classical Strömgren HII region. The parameters are valid inside the striped triangle in Fig. 5.

These high density clumps throughout the $\mathrm{H}$ II region close to Eta Carinae explain the presence of the strong Balmer absorption lines. They are exposed to the intense UV radiation of the central source, which, thanks to the three mechanisms considered above provides an overpopulation of metastable hydrogen atoms. In essence, measurement of these Balmer absorption lines provides a convenient diagnostic for detection of neutral clumps in an ionized medium and should be explored further, especially in consideration of the multiple lines arising from metastable lines of singly-ionized iron-peak elements.

In conclusion, the aim of the model presented in this paper was to interpret the presence of the narrow Balmer absorption 
lines seen in the spectra of $\eta$ Car and its surrounding nebulosities. We used average values of the hydrogen densities in its ground state and the excited metastable state, $N_{1}$ and $N_{2}$, respectively, to make a simple model of metastable $\mathrm{H}$ I in line of sight of background starlight in the form of broad P-Cygni Balmer line emission. The strength of the Balmer line absorption gives information about the column density of metastable hydrogen, but it does not reveal the actual radial dependence of $N_{2}$. More realistic models of the radial hydrogen distribution $N_{\mathrm{H}}(r)$ may provide means of estimating the total hydrogen mass in the equatorial region. However, this problem is beyond the scope of the present paper.

Acknowledgements. This paper is based upon observations made with the NASA/ESA Hubble Space Telescope, obtained at the Space Telescope Science Institute, which is operated by the Association of Universities for Research in Astronomy, Inc., under NASA contract NAS 5-26555. Funding was provided through the STIS Guaranteed Time Observations (GTO). V.S.L. acknowledges financial support through grants (S.J.) from the Royal Swedish Academy of Sciences and the Wenner-Gren Foundations, as well as Lund Observatory for hospitality and the Russian Foundation for Basic Research (grant No. 103-02-16377). The research project is supported by a grant (S.J.) from the Swedish National Space Board.

\section{References}

Damineli, A. 1996, ApJ, 460, L49

Davidson, K., Ebbets, D., Johansson, S., et al. 1997, AJ, 113, 335

Davidson, K., Ebbets, D., Weigelt, G., et al. 1995, AJ, 109, 1784

Davidson, K., \& Humphreys, R. M. 1997, ARA\&A, 35, 1

Davidson, K., Smith, N., Gull, T. R., Ishibashi, K., \& Hillier, D. J. 2001, AJ, 121, 1569

Davidson, K., Martin, J., Humphreys, R., et al. 2005, AJ, 129, 900

Gull, T., Ishibashi, K., Davidson, K., \& Collins, N. 2001, in Eta Carinae and Other Mysterious Stars: The Hidden Opportunities of Emission Spectroscopy, ASP Conf. Ser., 242, 391

Gull, T., Viera, G., Danks, A., et al. 2004, ApJ, 620, 442

Ishibashi, K., Gull, T. R., Davidson, K., et al. 2003, AJ, 125, 3222

Johansson, S., \& Letokhov, V. S. 2003, PASP, 115, 1375

Morris, P. W., Waters, L. B. F. M., Barlow, M. J., et al. 1999, Nature, 402, 502

Morse, J. A., Davidson, K., Bally, J., et al. 1998, AJ, 116, 2443

Pittard, J. M., \& Corcoran, M. F. 2002, A\&A, 383, 636

Smith, N., Davidson, K., Gull, T. R., Ishibashi, K., \& Hillier, D. J. 2003, ApJ, 586, 432

Smith, N., Morse, J., Gull, T. R., et al. 2004, ApJ, 610, 105

Verner, E., Bruhweiler, F., \& Gull, T. R. 2005, ApJ, in press

Weigelt, G., \& Ebersberger, J. 1986, A\&A, 163, L5

Zethson, T. 2001, Ph.D. Thesis, Lund University

Zethson, T., Johansson, S., Davidson, K., et al. 1999, A\&A, 344, 211 